TRANSACTIONS OF THE

AMERICAN MATHEMATICAL SOCIETY

Volume 363, Number 1, January 2011, Pages 143-164

S 0002-9947(2010)05348-6

Article electronically published on August 31, 2010

\title{
X-RAYS OF FORMS AND PROJECTIONS OF CURRENTS
}

\author{
BRUCE SOLOMON
}

\begin{abstract}
We study a new Radon-like transform that averages projected $p$ forms in $\mathbf{R}^{n}$ over affine $(n-k)$-spaces. We then prove an explicit inversion formula for our transform on the space of rapidly-decaying smooth $p$-forms. Our transform differs from the one in the work by Gelfand, Graev and Shapiro (1969). Moreover, if it can be extended to a somewhat larger space of $p$-forms, our inversion formula will allow the synthesis of any rapidly-decaying smooth $p$-form on $\mathbf{R}^{n}$ as a (continuous) superposition of pullbacks from $p$-forms on $k$-dimensional subspaces. In turn, such a synthesis implies an explicit formula (which we derive) for reconstructing compactly supported currents in $\mathbf{R}^{n}$ (e.g., compact oriented $k$-dimensional subvarieties) from their oriented projections onto $k$-planes.
\end{abstract}

\section{INTRODUCTION}

Question. Do the projections of an oriented $p$-dimensional object in $\mathbf{R}^{n}$ into $k$-dimensional subspaces - cancellation allowed - determine it?

Question. Can one use its projections to explicitly reconstruct the object?

Here, these questions prompt us to define a new integral transform of "Radon" type for differential $p$-forms, and to derive a formula that inverts it on the space of rapidly-decaying $p$-forms. Our inversion formula doesn't quite settle the questions above, but it comes very close: We believe it shows exactly what their answers have to be.

By no means are we first to construct a tomography of $p$-forms: In the late 1960s, Gelfand, Graev and Shapiro did so in GGS, and their work was followed by a series of related papers (e.g., GGG]). But our theory complements theirs, and unlike it, bears directly on the questions above.

To understand our transform, and see how it differs from the one in GGS, recall that any linear $k$-plane $P$, together with its orthogonal complement $P^{\prime}$, splits $\mathbf{R}^{n}$ as the direct sum $\mathbf{R}^{n}=P \oplus P^{\prime}$. The exterior algebras $\bigwedge_{*} P$ and $\bigwedge_{*} P^{\prime}$ then both form subspaces of the larger exterior algebra $\bigwedge_{*} \mathbf{R}^{n}$. Note, however, that their sum does not generally span $\bigwedge_{*} \mathbf{R}^{n}$.

Now consider a continuous $p$-form $\alpha$ in $\mathbf{R}^{n}$, and suppose it decays fast enough to make it integrable over any affine $(n-k)$-plane. Our transform assigns, to each $\xi \in P$, a value $\mathcal{R}_{k} \alpha(P, \xi) \in \bigwedge_{p} P$, by projecting $\alpha(x)$ into $\bigwedge_{p} P$ at each $x \in P^{\prime}$, and then integrating along $P^{\prime}$. We emphasize that $\mathcal{R}_{k} \alpha(P, \xi)$ thus belongs to $\bigwedge_{p} P$.

Received by the editors July 22, 2008.

2010 Mathematics Subject Classification. Primary 44A12, 42A85, 58A10, 58A25.

(C)2010 American Mathematical Society Reverts to public domain 28 years from publication 
Like ours, the transform in GGS integrates along $P^{\prime}$. But it does so after projecting $\alpha(x)$ into $\bigwedge_{p} P^{\prime}$, not $\bigwedge_{p} P$, at each $x \in P^{\prime}$. Accordingly, their transform assigns, to each $\xi \in P$, a value in $\bigwedge_{p} P^{\prime}$ instead of $\bigwedge_{p} P$. Though procedurally similar to ours, this process encodes very different information about the input $p$-form $\alpha$.

For instance, when $\alpha$ is a 1-form in $\mathbf{R}^{3}$, our 2-plane transform (which integrates along lines perpendicular to each linear 2-plane) is invertible, in the sense that we can recover any rapidly decaying 1 -form $\alpha$ from its transform $\mathcal{R}_{2} \alpha$. The transform in GGS, contrastingly, is not invertible in that case, for it annihilates all exact 1-forms. Indeed, its integration step computes standard line integrals, and the line integral of a rapidly decaying exact form must vanish.

Further, the low dimensions of the example just given oversimplify the distinction between our transform and that of [GGS]. For when $k=n-1=2$, and $p=1$, the inclusion

$$
\bigwedge_{p} P \oplus \bigwedge_{p} P^{\prime} \subset \bigwedge_{p} \mathbf{R}^{n}
$$

becomes an isomorphism. In that case, adding our transform to the one in GGS gives the classical (trivially vectorized) Radon transform. Roughly speaking, that puts any one of these transforms in the span of the other two, in which case our work produces nothing fundamentally new. But in general - in fact, whenever $k>p>$ 1 - the inclusion above is proper, and this makes our transform truly inequivalent to that of GGS.

To explain why our transform and inversion formulas are not just new, but also interesting, we revisit our opening questions. The oriented $p$-dimensional objects we have in mind there are compactly supported $p$-dimensional currents, in the sense of deRham. As we explain in $\S 6$, they belong to the dual of the space of smooth differential $p$-forms, and they project onto $k$-dimensional subspaces in a simple, natural way. Their relationship to $p$-forms makes the problem of reconstructing a current from its projections equivalent to that of writing any rapidly decaying $p$ form as a superposition of forms that are $k$-planar, that is, pullbacks of $p$-forms from $k$-dimensional subspaces via orthogonal projection. Formally, one gets precisely the right type of superposition formula from our inversion result, and when $p=0$ (where our whole theory reduces to that of the classical scalar Radon transform) this formula is known to hold. We feel sure that it holds for $p>0$ too, and state the expected facts, along with the answers they give to our opening questions, as explicit conjectures after proving our inversion result (Theorem 6.1). We hope that our work tempts someone having more skill with the required analytical tools to tie up these loose ends.

Meanwhile, discovering the appropriate tools, deriving the correct inversion formula, and proving its validity on the smaller space of rapidly decaying $p$-forms will occupy us here. Before beginning, we briefly preview our plan of attack.

Section 2 sets preliminary concepts and notation dealing mainly with exterior algebra and the Fourier transform. The material is routine, except possibly for Propositions 2.8 and 2.9, which show how the Fourier transform intertwines $d, \delta$, $d \delta$, and $\delta d$ with certain operations in the exterior algebra. These elegant formulae are easy, but we have not seen them elsewhere, and they combine to intertwine the half-Laplacians $d \delta$ and $\delta d$ with the projections we call $\Pi$ and $\Phi$ in $\Lambda_{*} \mathbf{R}^{n}$. We later exploit these facts to derive Corollary 6.2, a simpler "differential" statement of our inversion formula valid for even codimension. 
In 93 , we define our transform $\mathcal{R}_{k}$ and its dual $\mathcal{R}_{k}^{*}$. We work out a couple of examples along the way to clarify definitions and suggest the typical behavior of these operators.

In 44, we start the core work of our paper by deriving an explicit formula for the composition $\mathcal{R}_{k}^{*} \circ \mathcal{R}_{k}$ as a convolution (Theorem 4.5). This type of resultand its utility for inverting a Radon transform - were first noted by B. Fuglede in $\mathrm{Fu}$. Indeed, our formula reduces to Fuglede's result when the $p$-forms on which it operates are mere scalar functions $(p=0)$. But the precise generalization for $p>0$ requires a more sophisticated analysis focused on the action of the orthogonal group $\mathrm{O}(n)$ on the exterior algebra $\bigwedge_{*} \mathbf{R}^{n}$. The resulting convolution formula thus forms the first significant contribution of our paper.

The other really new element is our computation of the precise Fourier transform of $\mathcal{R}_{k}^{*} \circ \mathcal{R}_{k}$, obtained as Theorem [5.1 in $\$ 5$. Roughly speaking, we carry it out by expanding the convolution kernel from $\$ 4$ as a sum of quotients of spherical harmonics by powers of the radial distance $r$. We then use a well-known lemma from Stein $\mathrm{St}$ to compute the Fourier transforms of these quotients.

We conclude in $\$ 6$ by combining the convolution formula and our Fourier transform computation to invert $\mathcal{R}_{k}$ (Theorem 6.1). When the codimension $n-k$ is even, our inversion formula simplifies: The intertwining rules at the end of $\$ 2$ replace the Fourier transforms and projections there to simple linear combinations of the "half-Laplacians" $d \delta$ and $\delta d$. Corollary 6.2 states the precise result.

Finally, our Inversion Theorem leads quickly to the formal superposition result mentioned earlier: We state it as Conjecture 6.5, and then show that it would answer our opening questions. We present those answers too, as Conjecture 6.8.

We completed this project with sabbatical support from Indiana University, a Lady Davis fellowship at the Technion in Haifa, Israel, and extended hospitality from Stanford University. We deeply appreciate the assistance of all three institutions.

\section{Preliminaries}

For later reference, we record the basic notation and background here, mostly about the exterior algebra, the Fourier transform, and their interaction. The reader may prefer skipping to $\$ 3$ and return here for clarification when the need arises.

2.1. Subspaces and complements. Let $\mathrm{G}_{n, k}$ denote the compact Grassmann manifold of all $k$-planes in $\mathbf{R}^{n}$. We always denote the orthogonal complement of a linear $k$-plane $P \in \mathrm{G}_{n, k}$ by $P^{\prime}$, and we write $k^{\prime}$ for $\operatorname{dim} P^{\prime}=\operatorname{codim} P=n-k$.

2.2. Exterior algebra. We write $\bigwedge_{*} V$ for the exterior algebra of a finitedimensional vector space $V$. Chapter 1 of Federer's book $\mathrm{Fe}$ gives a careful (albeit terse) development of the exterior algebra from scratch. Here we review just a few relevant points.

First, $\bigwedge_{*} V$ has the direct sum decomposition

$$
\bigwedge_{*} V=\bigoplus_{k=0}^{n} \bigwedge_{k} V
$$

where $\bigwedge_{k} V$ denotes the subspace generated by $k$-fold wedge products of the form $v_{1} \wedge v_{2} \wedge \cdots \wedge v_{k}$, with $v_{i} \in V$. We call the elements of $\bigwedge_{k} V k$-vectors. 
Given an orthonormal basis $e_{1}, e_{2}, \ldots, e_{n}$ for $V$, we get a basis for $\bigwedge_{k} V$ comprising the $\left(\begin{array}{l}n \\ k\end{array}\right) \quad k$-vectors

$$
e_{\lambda}:=e_{\lambda_{1}} \wedge e_{\lambda_{2}} \wedge \cdots \wedge e_{\lambda_{k}}
$$

where $\lambda$ runs over all the increasing, length- $k$ subsequences of $\{1,2, \ldots, n\}$. Declaring this basis orthonormal extends the inner product on $V$ to an inner product $\langle\cdot, \cdot\rangle$ on $\bigwedge_{*} V$.

We get a linear transformation on $\bigwedge_{*} V$ by left-wedging with any fixed vector $v \in V$. We indicate the adjoint of this mapping (relative to the inner product above on $\bigwedge_{*} V$ ) by " $\vee v$ " and define it by requiring

$$
\langle\alpha \vee v, \beta\rangle:=\langle\alpha, v \wedge \beta\rangle
$$

for all $\alpha, \beta \in \bigwedge_{*} V$.

Any linear map $f: V \rightarrow W$ between vector spaces induces a multilinear extension between their respective exterior algebras via the simple formula

$$
v_{1} \wedge v_{2} \wedge \cdots \wedge v_{k} \longmapsto f\left(v_{1}\right) \wedge f\left(v_{2}\right) \wedge \cdots \wedge f\left(v_{k}\right) .
$$

Two examples particularly relevant for us arise with respect to a subspace $P \subset \mathbf{R}^{n}$ :

(1) Inclusion: The inclusion $f: P \rightarrow \mathbf{R}^{n}$ extends to an inclusion $\bigwedge_{*} P \subset$ $\bigwedge_{*} \mathbf{R}^{n}$. This lets us regard elements of $\bigwedge_{*} P$ as belonging to $\bigwedge_{*} \mathbf{R}^{n}$. We shall do so below routinely without further comment.

(2) Projection: The orthogonal projection $\mathbf{R}^{n} \rightarrow P$ is adjoint to the inclusion $P \subset \mathbf{R}^{n}$ with respect to the usual dot product. It extends to a projection $\bigwedge_{*} \mathbf{R}^{n} \rightarrow \bigwedge_{*} P$ that is orthogonal for the inner product $\langle\cdot, \cdot\rangle$ defined above on $\bigwedge_{*} \mathbf{R}^{n}$. We often suffix a vertical bar to indicate this projection. For instance, we write the projection of a vector $v$ on $\mathbf{R}^{n}$ to $P$ as $\left.v\right|_{P}$.

Let $H_{x}$ denote the hyperplane perpendicular to a non-zero $x \in \mathbf{R}^{n}$. The splitting $\mathbf{R}^{n}=H_{x} \oplus H_{x}^{\prime}$ induces a split in the exterior algebra:

$$
\bigwedge_{*} \mathbf{R}^{n}=\bigwedge_{*} H_{x} \oplus\left(x \wedge \bigwedge_{*} H_{x}\right) .
$$

We designate the projections onto the first and second factors here respectively by

$$
\Pi_{x}: \bigwedge_{*} \mathbf{R}^{n} \rightarrow \bigwedge_{*} H_{x} \quad \text { and } \quad \Phi_{x}: \bigwedge_{*} \mathbf{R}^{n} \rightarrow x \wedge \bigwedge_{*} H_{x} .
$$

The first of these is induced by the orthogonal projection $\mathbf{R}^{n} \rightarrow H_{x}$, as in item (2) above. We give it a special symbol nonetheless, as it plays a key role later on.

Finally, note that $\Pi_{x}$ acts as the identity (and $\Phi_{x}$ vanishes) on the degree-0 summand $\bigwedge_{0} \mathbf{R}^{n}$, because the latter lies entirely in $\bigwedge_{*} H_{x}$.

2.3. Differential forms. For our purposes, a differential $p$-form on a subspace $P \subset \mathbf{R}^{n}$ is a mapping $\phi: P \rightarrow \bigwedge_{p} \mathbf{R}^{n *}$, where $\mathbf{R}^{n *}$ denotes the dual of $\mathbf{R}^{n}$. The dot product identifies $\mathbf{R}^{n}$ with $\mathbf{R}^{n *}$ via

$$
\left(p_{1}, p_{2}, \ldots, p_{n}\right) \quad \leftrightarrow \quad p_{1} d x_{1}+p_{2} d x_{2}+\cdots+p_{n} d x_{n}
$$

and because of this isomorphism, we will frequently ignore the distinction between vectors and covectors. We often regard maps from $\mathbf{R}^{n}$ to $\bigwedge_{p} \mathbf{R}^{n}$ as differential forms, for instance.

Call a differential form $\phi$ on $\mathbf{R}^{n} k$-planar if we can write it as the "pullback" of a differential form $\psi$ native to some $k$-dimensional subspace $P \subset \mathbf{R}^{n}$ via the orthogonal projection $\mathbf{R}^{n} \rightarrow P$. Using the notation introduced above, this simply 
means that for some $P \in \mathrm{G}_{n, k}$ and all $x \in \mathbf{R}^{n}, \phi$ satisfies the following two conditions:

(1) $\phi(x)=\phi\left(\left.x\right|_{P}\right)$

(2) $\phi(x)=\left.\phi(x)\right|_{P}$.

For instance, the forms commonly denoted by $d x+d y$ and $x d x \wedge d y$ are both 2-planar on $\mathbf{R}^{3}$ with $P$ defined by $z=0$.

Contrastingly, neither $z d x \wedge d y$ nor $d x \wedge d y \wedge d z$ are 2-planar. The former satisfies condition (2) for the plane $z=0$, but not (1). The latter satisfies (1) but fails condition (2) for all $P \in \mathrm{G}_{2,3}$.

Remark 2.4. The point of Conjecture 6.5 is that we may construct many $q$-forms in $\mathbf{R}^{n}$ by averaging $k$-planar $q$-forms over $\mathrm{G}_{n, k}$.

Next, recall that we take the exterior derivative of a differential form $\phi$ on $\mathbf{R}^{n}$ using the formula

$$
d \phi=\sum_{i=1}^{n} d x_{i} \wedge \frac{\partial \phi}{\partial x_{i}} .
$$

Somewhat less familiar is the degree-decreasing divergence operator $\delta$. Our notation (cf. 2.2.2) allows us to express it thus:

$$
\delta \phi=\sum_{i=1}^{n} \frac{\partial \phi}{\partial x_{i}} \vee d x_{i} .
$$

Note that we omit the minus sign often used to define $\delta$ so that

$$
(d \delta+\delta d) \phi=\sum_{i=1}^{n} \frac{\partial^{2} \phi}{\partial x_{i}^{2}}=: \Delta \phi,
$$

making $\Delta$ denote the positive sum of pure second partials. In 2.7 we will see the Fourier transform relate $\delta d$ and $d \delta$ to the projections $\Pi$ and $\Phi$ of (2.2.4) above.

2.5. Mapping spaces. We will encounter tensor fields on $\mathbf{R}^{n}$, which, like differential forms, take values in a normed vector space $V$. To measure decay rates of these mappings near infinity, we define the seminorm $\|F\|_{(s)}$ for any map $F: \mathbf{R}^{n} \rightarrow V$, and any $s \geq 0$, via

$$
\|F\|_{(s)}:=\lim _{r \rightarrow \infty} \sup _{|x|>r}|x|^{s}|F(x)| .
$$

Assigning a finite value to $\|F\|_{(s)}$ is slightly more precise than saying " $F$ is $O\left(|x|^{-s}\right)$," which merely means $\|F\|_{(s)}<\infty$. Note that when $F$ is locally integrable, we ensure summability on any proper subspace of $\mathbf{R}^{n}$ - though not on $\mathbf{R}^{n}$ itself-by assuming $\|F\|_{(n)}<\infty$.

We will say that $F: \mathbf{R}^{n} \rightarrow V$ decays rapidly if $\|F\|_{(s)}<\infty$ for every $s \geq 0$, and we define the space $\mathcal{S}\left(\mathbf{R}^{n}, V\right)$ of Schwartz $V$-fields as the set of all smooth $F: \mathbf{R}^{n} \rightarrow V$ with rapidly decaying derivatives of all orders. To simplify notation, we will abbreviate

$$
\mathcal{S}\left(\mathbf{R}^{n}, V, W\right):=\mathcal{S}\left(\mathbf{R}^{n}, \operatorname{Hom}(V, W)\right),
$$

and when $P \subset \mathbf{R}^{n}$ is a subspace,

$$
\begin{aligned}
& \mathcal{S}_{p}(P):=\mathcal{S}\left(P, \bigwedge_{p} P\right) \text { (Schwartz } p \text {-forms), } \\
& \mathcal{S}(P):=\mathcal{S}_{0}(P)=\mathcal{S}(P, \mathbf{C}) \text { (Schwartz functions). }
\end{aligned}
$$


2.6. Tempered $(V, W)$-distributions. When $V$ and $W$ are inner-product spaces, we write $\mathcal{S}^{*}\left(\mathbf{R}^{n}, V, W\right)$ for the space of continuous linear functionals $\mathcal{S}\left(\mathbf{R}^{n}, V\right) \rightarrow W$, and call its constituent functionals tempered $(V, W)$ distributions or $\operatorname{Hom}(V, W)$-valued distributions, or, when the context allows, simply tempered distributions on $\mathbf{R}^{n}$. When applying such a distribution $\tau$ to a Schwartz $V$-field $F$, we write $\tau[F]$, with square brackets to emphasize the distributional context.

Certain distributions arise as locally integrable operator fields. An operator field is a mapping $T$ on $\mathbf{R}^{n}$ that assigns a linear transformation $T_{x} \in \operatorname{Hom}(V, W)$ to each $x \in \mathbf{R}^{n}$. When $T$ satisfies $\|T\|_{(p)}<\infty$ for some $p \geq 0$, and is also locally integrable, it represents a tempered $\operatorname{Hom}(V, W)$-valued distribution (which we still call $T$ ) via integration:

$$
T[F]:=\int_{\mathbf{R}^{n}} T(x) \cdot F(x) d x, \quad \text { for all } F \in \mathcal{S}\left(\mathbf{R}^{n}, V\right) .
$$

Here and henceforth the dot operator in " $A . X$ " instructs us to feed the vector $X \in V$ to the operator $A$. This will save us from over-nesting parentheses.

Equation (2.6.1) includes $\mathcal{S}\left(\mathbf{R}^{n}, V, W\right)$ into $\mathcal{S}^{*}\left(\mathbf{R}^{n}, V, W\right)$. Since the inner product identifies $V$ with its dual $V^{*}$, it also identifies $\mathcal{S}\left(\mathbf{R}^{n}, V\right)$ with $\mathcal{S}\left(\mathbf{R}^{n}, V^{*}\right)$, so by (2.6.1) also includes $\mathcal{S}\left(\mathbf{R}^{n}, V\right)$ into $\mathcal{S}^{*}\left(\mathbf{R}^{n}, V^{*}\right)$.

As in the scalar setting, one can extend, to all tempered $(V, W)$-distributions, many operators initially defined only on some subset that includes $\mathcal{S}\left(\mathbf{R}^{n}, V, W\right)$. The classic example is differentiation: When $T \in \mathcal{S}\left(\mathbf{R}^{n}, V, W\right)$, integration by parts gives

$$
\left(\frac{\partial T}{\partial x_{i}}\right)[F]=T\left[-\frac{\partial F}{\partial x_{i}}\right] .
$$

The right side above makes sense for any tempered $(V, W)$-distribution $T$, assuming $F \in \mathcal{S}\left(\mathbf{R}^{n}, V\right)$, so the identity above serves to define $\partial T / \partial x_{i}$ for any $T \in \mathcal{S}^{*}\left(\mathbf{R}^{n}, V, W\right)$.

2.7. Fourier transform. Using the normalizing conventions of Stein $\mathrm{St}$, we define the Fourier transform of any integrable $V$-field $F$ on $\mathbf{R}^{n}$ as the $V$-field given by

$$
\mathcal{F}(F)(\xi):=\int_{\mathbf{R}^{n}} F(x) e^{-2 \pi \mathrm{i} \xi \cdot x} d x .
$$

Any basic treatment (e.g. [Str] ) will establish fundamental properties of the scalar ( $V=\mathbf{C}$ ) Fourier transform which extend trivially to our vector-valued setting. These include:

(1) $\mathcal{F}$ maps $\mathcal{S}\left(\mathbf{R}^{n}, V\right)$ into itself.

(2) $\mathcal{F}$ is an $L^{2}$ isometry, and modulo a sign, inverts itself on $\mathcal{S}\left(\mathbf{R}^{n}, V\right)$. Specifically,

$$
\mathcal{F F}(F)=F^{-} .
$$

The superscript "_" here signals composition with reflection through the origin (cf. Appendix).

(3) Given $T \in \mathcal{S}\left(\mathbf{R}^{n}, V, W\right)$ and $F \in \mathcal{S}\left(\mathbf{R}^{n}, V\right), \mathcal{F}$ intertwines the convolution product $T \star F$, given by

$$
(T \star F)(y):=\int_{\mathbf{R}^{n}} T(y-x) \cdot F(x) d x,
$$


with the functional operation, in the sense that

$$
\mathcal{F}(T \star F)=\mathcal{F}(T) . \mathcal{F}(F) .
$$

The definition of convolution doesn't actually require the rapid decay of $T$ and $F$, but when both are Schwartz, so is $T \star F$, which allows one to extend the Fourier convolution rule to a distributional setting. We prove general statements of this type in our Appendix (Observations 7.1 and 7.2).

(4) $\mathcal{F}$ is "selfadjoint" in the sense that when $T \in \mathcal{S}\left(\mathbf{R}^{n}, V, W\right)$ and $F \in$ $\mathcal{S}\left(\mathbf{R}^{n}, V\right)$, we have

$$
\int_{\mathbf{R}^{n}} \mathcal{F}(T) \cdot F d x=\int_{\mathbf{R}^{n}} T \cdot \mathcal{F}(F) d x .
$$

Note that by using distributional notation, we can express this last identity as

$$
\mathcal{F}(T)[F]=T[\mathcal{F}(F)],
$$

which extends the Fourier transform to all of $\mathcal{S}^{*}\left(\mathbf{R}^{n}, V, W\right)$.

(5) Finally, recall the useful way that $\mathcal{F}$ intertwines differentiation and monomial multiplication:

For any $F \in \mathcal{S}\left(\mathbf{R}^{n}, V\right)$, we have the identities

$$
\mathcal{F}\left(\frac{\partial F}{\partial x_{j}}\right)=2 \pi \mathrm{i} \xi_{j} \mathcal{F}(F), \quad \frac{\partial}{\partial \xi_{j}} \mathcal{F}(F)=-2 \pi \mathrm{i} \mathcal{F}\left(x_{j} F\right) .
$$

These well-known identities follow directly from the definition of $\mathcal{F}$, but they imply less familiar rules for intertwining with the exterior derivative and divergence operators of (2.4.1) and (2.4.2):

Proposition 2.8. For any $\phi \in \mathcal{S}_{p}\left(\mathbf{R}^{n}\right)$, we have

$$
\begin{array}{rlrl}
\mathcal{F}(d \phi)=\mathrm{i} \pi d|\xi|^{2} \wedge \mathcal{F}(\phi), & \mathcal{F}(\delta \phi) & =\mathrm{i} \pi \mathcal{F}(\phi) \vee d|\xi|^{2}, \\
d \mathcal{F}(\phi)=-\mathrm{i} \pi \mathcal{F}\left(d|x|^{2} \wedge \phi\right), & \delta \mathcal{F}(\phi)=-\mathrm{i} \pi \mathcal{F}\left(\phi \vee d|x|^{2}\right) .
\end{array}
$$

Proof. Using $d|x|^{2}=2 \sum_{j} x_{j} d x_{j}$ and similarly for $d|\xi|^{2}$, the stated identities follow easily from (2.7.3) above, the adjoint identity (2.2.2) relating $\vee$ to $\wedge$, and the formulae for $d$ and $\delta$ given by (2.4.1) and (2.4.2).

Since $d|x|^{2}$ is dual to $2 x$, one easily combines the identities above with (2.2.2) to relate the "half-Laplacians" $d \delta$ and $\delta d(2.4 .3)$ to the projections $\Pi$ and $\Phi$ as promised at the end of $\$ 2.3$ above:

Proposition 2.9. Let $r:=|x|$ denote the radial distance function on $\mathbf{R}^{n}$. Then we have the following identities on $\mathcal{S}_{p}\left(\mathbf{R}^{n}\right)$ :

$$
d \delta=-4 \pi^{2} \mathcal{F}^{-1} \circ r^{2} \Phi \circ \mathcal{F}, \quad \delta d=-4 \pi^{2} \mathcal{F}^{-1} \circ r^{2} \Pi \circ \mathcal{F}
$$

and since $d \delta+\delta d=\Delta$,

$$
\Delta=-4 \pi^{2} \mathcal{F}^{-1} \circ r^{2} \circ \mathcal{F}
$$

We don't know any reference for the first two identities here, even though the last is common knowledge. 


\section{The transforms $\mathcal{R}_{k}$ And $\mathcal{R}_{k}^{*}$}

3.1. The transform. The transforms we describe in this section exchange differential forms on $\mathbf{R}^{n}$ with tensor fields on the canonical bundle $\Gamma_{n, k}$ over $\mathrm{G}_{n, k}$. We define the latter bundle via

$$
\Gamma_{n, k}:=\left\{(P, \xi) \in \mathrm{G}_{n, k} \times \mathbf{R}^{n}: \xi \in P\right\} .
$$

Definition 3.2 (The transform $\mathcal{R}_{k}$ ). Given a continuous differential $p$-form $\alpha$ on $\mathbf{R}^{n}$ with $\|\alpha\|_{(n)}<\infty$, we denote its transform by

$$
\mathcal{R}_{k} \alpha: \Gamma_{n, k} \rightarrow \bigwedge_{p} \mathbf{R}^{n}
$$

and define it as an integral:

$$
\mathcal{R}_{k} \alpha(P, \xi):=\left.\int_{P^{\prime}} \alpha(\xi+\eta)\right|_{P} d \eta
$$

Remark 3.3. When $p=0$, so that $\alpha$ is just a scalar-valued function, we have $\left.\alpha\right|_{P}=\alpha$. In this case, $\mathcal{R}_{k}$ reduces to the classical $k^{\prime}$-plane transform (cf. $[\mathrm{H}$, p. 28]).

Remark 3.4. The definition above characterizes $\mathcal{R}_{k} \alpha$ as a mapping from $\Gamma_{n, k}$ to $\bigwedge_{*} \mathbf{R}^{n}$, making it a section of the trivial $\bigwedge_{*} \mathbf{R}^{n}$ bundle over $\Gamma_{n, k}$. Actually, $\mathcal{R}_{k} \alpha$ takes values in the subbundle whose fiber over $(P, \xi)$ is the pullback subspace $\bigwedge_{p} P \subset \bigwedge_{p} \mathbf{R}^{n}$.

Remark 3.5. The integral above does not signify oriented integration of forms in the Stokes-theoretic sense. For one thing, the integrand belongs pointwise to $\bigwedge_{p} P$, hence vanishes on any $k^{\prime}$-tuple of vectors tangent to the domain of integration $P^{\prime}$. Rather, the integral performs elementary vector integration of a $\bigwedge_{p} P$-valued function against the Lebesgue measure on $P^{\prime}$. The following example illustrates this.

Example 3.6. Suppose $a \in \bigwedge_{p} \mathbf{R}^{n}$, let $\lambda>0$, and define the $p$-form $\phi(x):=$ $e^{-\lambda|x|^{2}} a$. We compute $\mathcal{R}_{k} \phi$ using the well-known identity

$$
\int_{\mathbf{R}^{n}} e^{-\lambda|x|^{2}} d x=\left(\frac{\pi}{\lambda}\right)^{n / 2}
$$

which implies, for any $(P, \xi) \in \Gamma_{n, k}$, that

$$
\int_{P^{\prime}} e^{-\lambda|\xi+\eta|^{2}} d \eta=\int_{P^{\prime}} e^{-\lambda|\xi|^{2}} e^{-\lambda|\eta|^{2}} d \eta=\left(\frac{\pi}{\lambda}\right)^{k^{\prime} / 2} e^{-\lambda|\xi|^{2}},
$$

whence

$$
\mathcal{R}_{k} \phi(P, \xi)=\left.\int_{P^{\prime}} e^{-\lambda|\xi+\eta|^{2}} a\right|_{P} d \eta=\left.\left(\frac{\pi}{\lambda}\right)^{k^{\prime} / 2} e^{-\lambda|\xi|^{2}} a\right|_{P} .
$$

The input $p$-form $\phi$ above decays rapidly, and on each fiber of $\Gamma_{n, k}$, its transform $\mathcal{R}_{k} \phi$ has the same property. The proposition below shows that this is no accident.

Let $\left|\mathbf{S}^{n}\right|$ denote the $n$-dimensional volume of the unit sphere in $\mathbf{R}^{n+1}$. 
Proposition 3.7. Suppose $\alpha$ is a p-form on $\mathbf{R}^{n}$ and $s \geq n$. Then for any $P \in \mathrm{G}_{n, k}$, we have

$$
\left\|\mathcal{R}_{k} \alpha(P, \cdot)\right\|_{\left(s-k^{\prime}\right)}<4\left|\mathbf{S}^{k^{\prime}-1}\right|\|\alpha\|_{(s)} .
$$

Proof. Without loss of generality, assume $\|\alpha\|_{(s)}<\infty$ for some $s \geq n$. Then for sufficiently large $\xi$ and any $\eta \in P^{\prime}$, we have

$$
|\alpha(\xi+\eta)|<2\|\alpha\|_{(s)}\left(1+|\xi|^{2}+|\eta|^{2}\right)^{-s / 2},
$$

hence also

$$
\begin{aligned}
\left|\mathcal{R}_{k} \alpha(P, \xi)\right| & \leq 2\|\alpha\|_{(s)} \int_{P^{\prime}}\left(1+|\xi|^{2}+|\eta|^{2}\right)^{-s / 2} d \eta \\
& \leq 2\|\alpha\|_{(s)}\left(1+|\xi|^{2}\right)^{-s / 2} \int_{P^{\prime}}\left(1+\frac{|\eta|^{2}}{1+|\xi|^{2}}\right)^{-s / 2} d \eta .
\end{aligned}
$$

Switching to polar coordinates and changing the radial variable $r=|\eta|$ to $\rho=$ $r / \sqrt{1+|\xi|^{2}}$, we now get

$$
\left|\mathcal{R}_{k} \alpha(P, \xi)\right| \leq 2\|\alpha\|_{(s)}\left|\mathbf{S}^{k^{\prime}-1}\right|\left(1+|\xi|^{2}\right)^{\frac{k^{\prime}-s}{2}} \int_{0}^{\infty}\left(1+\rho^{2}\right)^{-s / 2} \rho^{k^{\prime}-1} d \rho .
$$

But

$$
\begin{aligned}
\int_{0}^{\infty}\left(1+\rho^{2}\right)^{-s / 2} \rho^{k^{\prime}-1} d \rho & <1+\int_{1}^{\infty}\left(1+\rho^{2}\right)^{-s / 2} \rho^{k^{\prime}-1} d \rho \\
& <1+\int_{1}^{\infty} \rho^{-s+k^{\prime}-1} d \rho,
\end{aligned}
$$

and $-s+k^{\prime}-1 \leq-n+k^{\prime}-1=-k-1<-1$, because $s \geq n$. The last integral above is therefore bounded by $1 / k \leq 1$.

3.8. The dual transform $\mathcal{R}^{*}$. As in the classical Radon theory, our transform $\mathcal{R}_{k}$ has a formal $L^{2}$ dual. In our situation, the dual sends continuous $\bigwedge_{p} \mathbf{R}^{n}$-valued fields on $\Gamma_{n, k}$ back to $p$-forms on $\mathbf{R}^{n}$.

Recall that the transitive action of $\mathrm{O}(n)$ determines a unique Haar probability measure on $\mathrm{G}_{n, k}$. We compute all integrals over $\mathrm{G}_{n, k}$ using that measure.

Definition 3.9. The dual transform $\mathcal{R}_{k}^{*}$ takes a continuous mapping $\beta: \Gamma_{n, k} \rightarrow$ $\bigwedge_{p} \mathbf{R}^{n}$ to the $p$-form on $\mathbf{R}^{n}$ given by

$$
\mathcal{R}_{k}^{*} \beta(x):=\left.\int_{\mathrm{G}_{n, k}} \beta(P, P x)\right|_{P} d P .
$$

Here $P x$ denotes the orthogonal projection of $x$ onto $P$.

Note that we always have

$$
\mathcal{R}_{k}^{*} \beta=\mathcal{R}_{k}^{*} \bar{\beta} \quad \text { when }\left.\quad(\bar{\beta}(P, \cdot)-\beta(P, \cdot))\right|_{P} \equiv 0 .
$$

For each $x \in \mathbf{R}^{n}$, the map $P \mapsto(P, P x)$ embeds $\mathrm{G}_{n, k}$ into $\Gamma_{n, k}$. The compactness of $\mathrm{G}_{n, k}$ and the continuity of $\beta$ therefore guarantee the existence of the integral defining $\mathcal{R}_{k}^{*}$.

Remark 3.10. Note too that for each fixed $P \in \mathrm{G}_{n, k}$, the integrand $\left.\beta(P, P x)\right|_{P}$ specifies a $k$-planar $p$-form on $\mathbf{R}^{n}$. It follows that anything in the image of $\mathcal{R}_{k}^{*}$ is a superposition, over all $P \in \mathrm{G}_{n, k}$, of $k$-planar $p$-forms. The statement of Conjecture 6.5 depends on this fact. 
Example 3.11. Fixing an arbitary $v \in \mathbf{R}^{3}$, let us compute the dual transform $\mathcal{R}_{2}^{*} \beta$ of the simple field $\beta: \Gamma_{3,2} \rightarrow \bigwedge_{1} \mathbf{R}^{3}$ given by

$$
\beta(P, \xi):=e^{-|\xi|^{2}} v .
$$

By definition,

$$
\mathcal{R}_{2}^{*} \beta(x)=\left.\int_{\mathrm{G}_{2,3}} \beta(P, P x)\right|_{P} d P=\left.\frac{1}{4 \pi} \int_{\mathbf{S}^{2}} e^{-\left|\omega^{\perp} x\right|^{2}} v\right|_{\omega^{\perp}} d \omega .
$$

Here $\omega^{\perp}$ denotes the plane orthogonal to $\omega \in \mathbf{S}^{2}$, and we have used the fact that $\mathbf{S}^{2}$ double-covers $\mathrm{G}_{3,2}$ homogeneously.

Note also that by virtue of Example 3.6 and (3.9.1), we have

$$
\mathcal{R}_{2}^{*} \beta=\mathcal{R}_{2}^{*} \mathcal{R}_{2} \phi,
$$

where $\phi$ is the 1 -form on $\mathbf{R}^{3}$ given by $\phi(x)=\pi^{-1 / 2} e^{-|x|^{2}} v$.

Write $r=|x|, u=x / r$, parametrize the unit circle perpendicular to $u$ with unit speed as $u^{\perp}(\theta)$, and put " $u$-polar" coordinates on $\mathbf{S}^{2}$ by setting

$$
\omega=\omega_{\lambda, \theta}:=\lambda u+\sqrt{1-\lambda^{2}} u_{\theta}^{\perp} .
$$

Then $\left|\omega^{\perp} x\right|^{2}=r^{2}|\omega-(\omega \cdot u) u|^{2}=r^{2}\left(1-\lambda^{2}\right)$, and hence

$$
\begin{aligned}
\mathcal{R}_{2}^{*} \beta(x) & =\frac{1}{4 \pi} \int_{-1}^{1} \int_{0}^{2 \pi} e^{-r^{2}\left(1-\lambda^{2}\right)}\left(v-\left(v \cdot \omega_{\lambda, \theta}\right) \omega_{\lambda, \theta}\right) d \theta d \lambda \\
& \left.=\frac{e^{-r^{2}}}{2 \pi} \int_{0}^{1} e^{r^{2} \lambda^{2}}\left(2 \pi v-\int_{0}^{2 \pi}\left(v \cdot \omega_{\lambda, \theta}\right) \omega_{\lambda, \theta}\right) d \theta\right) d \lambda,
\end{aligned}
$$

thanks to the symmetry of the integrand around $\lambda=0$.

Now split $v$ as $v=v^{\|}+v^{\perp}$, with $v^{\|}$parallel to $u$, and $v^{\perp} \cdot u=0$. A routine exercise then finds the parenthesized part of the integrand above to be

$$
\pi\left(1+\lambda^{2}\right) v^{\perp}+2 \pi\left(1-\lambda^{2}\right) v^{\|} .
$$

Put this into the last integral for $\mathcal{R}_{2}^{*} \beta$ above, and make the substitution $s:=r \lambda$ to get

where

$$
\mathcal{R}_{2} \beta(x)=\frac{1}{2} \mathcal{I}_{+}(r) v^{\perp}+\mathcal{I}_{-}(r) v^{\|},
$$

$$
\mathcal{I}_{ \pm}(r)=r^{-3} e^{-r^{2}} \int_{0}^{r} e^{s^{2}}\left(r^{2} \pm s^{2}\right) d s
$$

Both $\mathcal{I}_{+}$and $\mathcal{I}_{-}$are smooth and decrease to zero as $r \rightarrow \infty$. Specifically, it is not hard to show, using l'Hôspital's rule, that

$$
\begin{aligned}
\lim _{r \rightarrow \infty} r^{2} \mathcal{I}_{+}(r) & =1, \\
\lim _{r \rightarrow \infty} r^{4} \mathcal{I}_{-}(r) & =1 / 2 .
\end{aligned}
$$

Putting these facts together with (3.11.1), we now see that

$$
\mathcal{R}_{2}^{*} \beta=\mathcal{R}_{2}^{*} \mathcal{R}_{2} \phi \sim \pi\left(\frac{v^{\perp}}{r^{2}}+\frac{v^{\|}}{r^{4}}\right) \quad \text { as } r \rightarrow \infty .
$$

In particular, we get decay, but not rapid decay, despite the rapid decay of the input field $\beta$. This is typical, as will follow from the convolution formula (Prop. 4.5). In this regard, the dual transform $\mathcal{R}_{k}^{*}$ behaves quite differently from the forward transform $\mathcal{R}_{k}$. 


\section{Convolution formula}

We now want to show that the composition $\mathcal{R}_{k}^{*} \circ \mathcal{R}_{k}$, applied to any locally integrable and suitably decaying $p$-form on $\mathbf{R}^{n}$, convolves that $p$-form with $|x|^{-k} \Pi$, up to some dimensional constant. As noted in our introduction, this fact generalizes the classical result of Fuglede $[\mathrm{Fu}$.

Recall (\$2.2) that $\Pi: \mathbf{R}^{n} \rightarrow \operatorname{Hom}\left(\bigwedge_{*} \mathbf{R}^{n}\right)$ is the operator field we get on $\mathbf{R}^{n}$ by mapping each non-zero $x \in \mathbf{R}^{n}$ to the orthogonal projection $\Pi_{x}: \mathbf{R}^{n} \rightarrow \bigwedge_{*} H_{x}$, where $H_{x}$ is the hyperplane perpendicular to $x$.

The Grassmannian $\mathrm{G}_{n, k}$ and the unit sphere $\mathbf{S}^{n-1} \subset \mathbf{R}^{n}$ are both homogeneous spaces of $\mathrm{O}(n)$. To rewrite $\mathcal{R}_{k}^{*} \circ \mathcal{R}_{k}$ as a convolution, we will first pull integrals over the Grassmannian back to integrals over $\mathrm{O}(n)$, and then push them down to $\mathbf{S}^{n-1}$. The lemmas below allow us to do so with precision.

4.1. Two averaging lemmas. The orthogonal group $\mathrm{O}(n)$ acts on $\mathbf{R}^{n}$ (and thus also on $\mathrm{G}_{n, k}$ ) by left-multiplication. For any $a \in \mathbf{S}^{n-1}$, denote the $a$-stabilizing subgroup of $\mathrm{O}(n)$ by $K_{a} \approx \mathrm{O}(n-1)$. Then $\mathrm{O}(n)$ is foliated by the mutually isometric left cosets of $K_{a}$, and if $\gamma \in \mathrm{O}(n)$, with $b:=\gamma a$, we define the coset

$$
K_{a b}:=\gamma K_{a}=K_{b} \gamma=\{\kappa \in \mathrm{O}(n): \kappa a=b\} .
$$

Note that $\mathrm{O}(n)$ has a left-invariant metric whose Hausdorff measure assigns total mass 1 to both $\mathrm{O}(n)$ and $K_{a}$ - hence also to each coset $K_{a b}$-in the appropriate dimensions. For integration, we always use these measures.

We have adapted the first formula below from Helgason [H, I (15)]. We need the second one to handle differential forms, as opposed to the scalar-valued functions treated there.

Lemma 4.2. Suppose $\phi$ maps $\mathrm{G}_{n, k}$ continuously into a vector space $V$. Then for any fixed $Q \in \mathrm{G}_{n, k}$, we have

$$
\int_{\mathrm{G}_{n, k}} \phi(P) d P=\int_{\mathrm{O}(n)} \phi(\gamma Q) d \gamma .
$$

Further, if $a \in \mathbf{S}^{n-1}$ and $\psi: \mathrm{O}(n) \rightarrow V$ is continuous, then

$$
\int_{\mathrm{O}(n)} \psi(\gamma) d \gamma=\frac{1}{\left|\mathbf{S}^{n-1}\right|} \int_{\mathbf{S}^{n-1}}\left(\int_{K_{a b}} \psi(\kappa) d \kappa\right) d b
$$

Here $d b$ and $d \kappa$ denote the Hausdorff measures on $\mathbf{S}^{n-1}$ and $K_{a}$, respectively.

Proof. Choose an arbitary $k$-plane $P \in \mathrm{G}_{n, k}$, and map $\mathrm{O}(n) \stackrel{e_{P}}{\longrightarrow} \mathrm{G}_{n, k}$ by defining $e_{P}(\gamma):=\gamma P$. Then $e_{P}(g \gamma)=g e_{P}(\gamma)$ for all $g, \gamma \in \mathrm{O}(n)$, and hence $e_{P}$ pushes the invariant probability measure on $\mathrm{O}(n)$ down to the one on $\mathrm{G}_{n, k}$. This lets us integrate $\phi$ over $\mathrm{G}_{n, k}$ relative to the latter measure by integrating $\phi \circ e_{P}$ relative to the former. The first formula asserts nothing more than that.

To get the second formula, we similarly map $\mathrm{O}(n) \stackrel{e_{a}}{\longrightarrow} \mathbf{S}^{n-1}$ via $e_{a}(\gamma):=\gamma a$. Then for any $b \in \mathbf{S}^{n-1}$, we have $e_{a}^{-1}(b)=K_{a b}$, and the coarea formula [Fe, 3.2.22] yields

$$
\int_{\mathrm{O}(n)} \psi(\gamma) J e_{a} d \gamma=\int_{\mathbf{S}^{n-1}}\left(\int_{K_{a b}} \psi(\kappa) d \kappa\right) d \gamma,
$$

with $J e_{a}$ denoting the Jacobian of $e_{a}$. But this Jacobian must be constant, because $e_{a}$ commutes with the transitive left $\mathrm{O}(n)$ action on itself. Set $\psi \equiv 1$ to see that $J e_{a}=\left|\mathbf{S}^{n-1}\right|$. 
Notation. We write $\mathrm{Ch}(n, m)$ as a horizontal alternative to the usual vertical symbol for binomial coefficients:

$$
\mathrm{Ch}(n, m):=\left(\begin{array}{c}
n \\
m
\end{array}\right)=\frac{n !}{m !(n-m) !} .
$$

Lemma 4.3. Suppose $P \in \mathrm{G}_{n, k}, x \in P^{\prime}$ and $H_{x}$ is the hyperplane perpendicular to $x$ (cf. \$2.2). Then for any $\alpha \in \bigwedge_{p} \mathbf{R}^{n}$ with $p \leq k$, we have

$$
\left.\int_{K_{x}} \alpha\right|_{\kappa P} d \kappa=\left.\frac{\mathrm{Ch}(k, p)}{\operatorname{Ch}(n-1, p)} \alpha\right|_{H_{x}} .
$$

Proof. Let $A$ denote the "averaging" operator $\left.\alpha \mapsto \int_{K_{x}} \alpha\right|_{\kappa P} d \kappa$ on the lefthand side of the formula above. Restriction to a subspace (followed by inclusion in $\bigwedge_{p} \mathbf{R}^{n}$; see 2.2 (11) above) is a symmetric endomorphism of $\bigwedge_{p} \mathbf{R}^{n}$, so $A$ is symmetric too.

Now observe that the action of $K_{x}$ on $\bigwedge_{p} \mathbf{R}^{n}$ respects the splitting (2.2.3); namely,

$$
\bigwedge_{p} \mathbf{R}^{n}=\bigwedge_{p} H_{x} \oplus\left(x \wedge \bigwedge_{p-1} H_{x}\right)
$$

It follows that $A$ commutes with this action. But $K_{x}$ is the full orthogonal group of $H_{x}$. So it acts transitively on both $p$ - and $(p-1)$-dimensional subspaces of $H_{x}$, hence irreducibly on both $\bigwedge_{p} H_{x}$ and $x \wedge \bigwedge_{p-1} H_{x}$. This makes each summand in the splitting above an eigenspace of $A$, so that in the notation of 2.2 , we have

$$
A=\lambda \Pi_{x}+\mu \Phi_{x}
$$

for some $\lambda, \mu \in \mathbf{R}$. But the definition of $A$ makes it clear that $\mu=0$, which, modulo the particular value of $\lambda$, proves our lemma.

To evaluate $\lambda$, observe that for any subspace $V \subset \mathbf{R}^{n}$, the projection $\bigwedge_{p} \mathbf{R}^{n} \rightarrow$ $\bigwedge_{p} V$ has trace given by

$$
\operatorname{tr}\left(\left.\cdot\right|_{V}\right)=\operatorname{dim} \bigwedge_{p} V=\left(\begin{array}{c}
\operatorname{dim} V \\
p
\end{array}\right)
$$

on $\bigwedge_{p} \mathbf{R}^{n}$. Taking the trace on both sides of (4.3.1) then gives

$$
\operatorname{tr}(A)=\lambda\left(\begin{array}{c}
n-1 \\
p
\end{array}\right)
$$

At the same time the definition of $A$ gives

$$
\operatorname{tr}(A)=\int_{K_{x}} \operatorname{tr}\left(\left.\cdot\right|_{\kappa P}\right) d \kappa=\left(\begin{array}{c}
k \\
p
\end{array}\right) .
$$

These two facts determine $\lambda$ and prove the lemma.

4.4. Convolution formula. We can now present the main result of this section:

Proposition 4.5 (Convolution formula). Given a locally integrable $p$-form $\alpha$ on $\mathbf{R}^{n}$ with $0 \leq p \leq k<n$ and $\|\alpha\|_{(n)}<\infty$, the convolution $\left(r^{-k} \Pi\right) \star \alpha$ exists, and we have

$$
\mathcal{R}_{k}^{*} \mathcal{R}_{k} \alpha=\frac{\left|\mathbf{S}^{k^{\prime}-1}\right| \operatorname{Ch}\left(k^{\prime}, p\right)}{\left|\mathbf{S}^{n-1}\right| \operatorname{Ch}(n-1, p)}\left(r^{-k} \Pi\right) \star \alpha .
$$


Proof. Our assumptions ensure that for each fixed $x \in \mathbf{R}^{n}$, the $p$-form

$$
y \longmapsto|y|^{-k} \Pi_{y} \alpha(y-x)
$$

is locally integrable and $O\left(|y|^{-n-k}\right)$ near infinity. This guarantees existence of the convolution.

To see that the convolution encodes $\mathcal{R}_{k}^{*} \mathcal{R}_{k}$ as claimed, combine the definitions of $\mathcal{R}_{k}$ and $\mathcal{R}_{k}^{*}$ with the fact that for any $x \in \mathbf{R}^{n}$ and $P \in \mathrm{G}_{n, k}$, we have $P x+P^{\prime}=x+P^{\prime}$ as affine subspaces. It follows easily that

$$
\left(\mathcal{R}_{k}^{*} \circ \mathcal{R}_{k}\right) \alpha(x)=\left.\int_{\mathrm{G}_{n, k}} \int_{P^{\prime}} \alpha(x+\eta)\right|_{P} d \eta d P .
$$

Now fix an arbitrary $Q \in \mathrm{G}_{n, k}$ and apply the first formula in Lemma 4.2, Then apply Fubini. Since $\gamma Q^{\prime}=\left\{\gamma \eta: \eta \in Q^{\prime}\right\}$ for any $\gamma \in \mathrm{O}(n)$, this gives

$$
\left(\mathcal{R}_{k}^{*} \circ \mathcal{R}_{k}\right) \alpha(x)=\left.\int_{Q^{\prime}} \int_{\mathrm{O}(n)} \alpha(x+\gamma \eta)\right|_{\gamma Q} d \gamma d \eta
$$

Note that when $\alpha$ has degree $p=0$, it takes mere scalar values, and the restriction to $\gamma Q$ has no effect. In that case, the inner integral reduces to a simple $x$-centered spherical average of $\alpha$, and the convolution formula quickly follows (cf. $[\mathrm{H}$, Ch.I $(34)]$ ). Because we assume $p>0$, however, the restriction complicates our task and we must now sort that out.

Start by using the second identity from Lemma 4.2. If we define $\bar{\eta}=\eta /|\eta|$, and recall from (4.1.1) that $K_{\bar{\eta} q^{\prime}}=K_{q^{\prime}} \gamma$ when $\gamma \in \mathrm{O}(n)$ satisfies $\gamma \bar{\eta}=q^{\prime}$, then (4.5.1) is rewritten as

$$
\begin{aligned}
\mathcal{R}_{k}^{*} \mathcal{R}_{k} \alpha(x) & =\frac{1}{\left|\mathbf{S}^{n-1}\right|} \int_{Q^{\prime}} \int_{\mathbf{S}^{n-1}}\left(\left.\int_{K_{\bar{\eta} q^{\prime}}} \alpha\left(x+|\eta| q^{\prime}\right)\right|_{\kappa Q} d \kappa\right) d q^{\prime} d \eta \\
& =\frac{1}{\left|\mathbf{S}^{n-1}\right|} \int_{Q^{\prime}} \int_{\mathbf{S}^{n-1}}\left(\left.\int_{K_{q^{\prime}}} \alpha\left(x+|\eta| q^{\prime}\right)\right|_{\kappa \gamma Q} d \kappa\right) d q^{\prime} d \eta
\end{aligned}
$$

where we have fixed some $\gamma \in K_{\bar{\eta} q^{\prime}}$. Since $\gamma Q$ is a $k$-dimensional subspace of $T_{q^{\prime}} \mathbf{S}^{n-1}$, Lemma 4.3 now applies to give

$$
\begin{aligned}
\mathcal{R}_{k}^{*} \mathcal{R}_{k} \alpha(x) & =\frac{1}{\left|\mathbf{S}^{n-1}\right|} \int_{Q^{\prime}} \int_{\mathbf{S}^{n-1}}\left(\left.\int_{K_{\bar{\eta}}} \alpha\left(x+|\eta| q^{\prime}\right)\right|_{\kappa \gamma Q} d \kappa\right) d q^{\prime} d \eta \\
& =\left.\frac{\operatorname{Ch}\left(k^{\prime}, p\right)}{\left|\mathbf{S}^{n-1}\right| \operatorname{Ch}(n-1, p)} \int_{Q^{\prime}} \int_{\mathbf{S}^{n-1}} \alpha\left(x+|\eta| q^{\prime}\right)\right|_{H_{q^{\prime}}} d q^{\prime} d \eta \\
& =\left.\frac{\left|\mathbf{S}^{k^{\prime}-1}\right| \operatorname{Ch}\left(k^{\prime}, p\right)}{\left|\mathbf{S}^{n-1}\right| \operatorname{Ch}(n-1, p)} \int_{0}^{\infty} \int_{\mathbf{S}^{n-1}} \alpha\left(x+r q^{\prime}\right)\right|_{H_{q^{\prime}}} r^{k^{\prime}-1} d q^{\prime} d r,
\end{aligned}
$$

switching from rectangular to polar coordinates on $Q^{\prime}$, with $r:=|\eta|$. But by writing $r^{k^{\prime}-1}=r^{n-1} r^{-k}, y:=-r q^{\prime}$, and noting that $H_{y}=H_{q^{\prime}}$, we can make the reverse move on $\mathbf{R}^{n}$ to deduce

$$
\mathcal{R}_{k}^{*} \mathcal{R}_{k} \alpha(x)=\left.\frac{\left|\mathbf{S}^{k^{\prime}-1}\right| \operatorname{Ch}\left(k^{\prime}, p\right)}{\left|\mathbf{S}^{n-1}\right| \operatorname{Ch}(n-1, p)} \int_{\mathbf{R}^{n}} \alpha(x-y)\right|_{H_{y}} r^{-k} d y .
$$


Now simply recall from the end of $\$ 2.2$ that restriction to $H_{y}$ induces the projection we call $\Pi_{y}$, so that

$$
\left.\alpha(x-y)\right|_{H_{y}} r^{-k}=|y|^{-k} \Pi_{y} \alpha(x-y) .
$$

Putting this into the equation above, we obtain our convolution formula.

Remark 4.6. Though we don't include the proof here, the convolution above must decay near infinity. To be precise, one can show that as $r:=|x| \rightarrow \infty$,

$$
\left|\left(r^{-k} T\right) \star f(x)\right|= \begin{cases}O\left(r^{-k}\right), & \|f\|_{L^{1}}<\infty, \\ O\left(r^{-k} \ln r\right), & \|f\|_{L^{1}}=\infty,\end{cases}
$$

for any locally integrable, vector-valued mapping $f$ on $\mathbf{R}^{n}$, assuming that $k<n$, $\|f\|_{(n)}<\infty$, and $T$ is a bounded operator field.

Remark 4.7. As noted in Remark 3.3, our transform reduces to the classical Radon $k^{\prime}$-plane transform when $p=0$. The reader will easily check that when we apply our convolution formula to any continuous 0 -form $f: \mathbf{R}^{n} \rightarrow \mathbf{R}$ with $\|f\|_{(n)}<\infty$, it too reduces to a known result (cf. [H, p. 29 (55)]), namely

$$
\mathcal{R}_{k}^{*} \circ \mathcal{R}_{k} f=\frac{\left|\mathbf{S}^{k^{\prime}-1}\right|}{\left|\mathbf{S}^{n-1}\right|} r^{-k} \star f .
$$

Remark 4.8. Roughly speaking, $\mathcal{R}_{k}^{*} \mathcal{R}_{k} \alpha$ will be at least as smooth as $\alpha$ itself. For when $\alpha$ has continuous derivatives $D^{\gamma} \alpha$ for all multi-indices $|\gamma| \leq q$, with $\left|D^{\gamma} \alpha\right|=O\left(r^{-n}\right)$, one easily checks that

$$
D^{\gamma}\left(\left(r^{-k} \Pi\right) \star \alpha\right)=\left(r^{-k} \Pi\right) \star D^{\gamma} \alpha,
$$

so that by our convolution formula, $\mathcal{R}_{k}^{*} \mathcal{R}_{k} \alpha$ is differentiable through order at least $q$ too.

\section{KERNELS}

To fully exploit the convolution formula, we also need to know the Fourier transform of its kernel $r^{-k} \Pi$. We assume $k<n$, so the singularity at $r=0$ is integrable, and $r^{-k} \Pi$ represents a tempered distribution. It therefore has a Fourier transform. Explicitly,

Theorem 5.1. As an operator on rapidly-decreasing $p$-forms, with $0 \leq p \leq k<n$, we have

$$
\mathcal{F}\left(r^{-k} \Pi\right)=\frac{1}{k} \frac{\left|\mathbf{S}^{k-1}\right|}{\left|\mathbf{S}^{k^{\prime}-1}\right|} \frac{(k-p) \Pi+(n-p) \Phi}{r^{k^{\prime}}} .
$$

When $p<k$, this makes $\mathcal{F}\left(r^{-k} \Pi\right)$ pointwise invertible, with

$$
\mathcal{F}\left(r^{-k} \Pi\right)^{-1}=\frac{\left|\mathbf{S}^{k^{\prime}-1}\right|}{\left|\mathbf{S}^{k-1}\right|} k r^{k^{\prime}}\left(\frac{\Pi}{k-p}+\frac{\Phi}{n-p}\right) .
$$

To prove this, we require two main ingredients. The first is Lemma 5.2 below, a beautiful formula that Stein bases on an identity he credits to Hecke [St, p.73]. The second, Lemma 5.5. prepares us to exploit Stein's lemma by expanding $\Pi$ as a sum involving spherical harmonics. 
Lemma 5.2. Suppose $h_{d}: \mathbf{R}^{n} \rightarrow \mathbf{C}$ is a homogeneous harmonic polynomial of degree $d$, and $0<k<n$. Then $r^{-d-k} h_{d}$ and its Fourier transform are both tempered distributions, with

$$
\mathcal{F}\left(r^{-d-k} h_{d}\right)=\mathrm{i}^{d} \frac{\left|\mathbf{S}^{d+k-1}\right|}{\left|\mathbf{S}^{d+k^{\prime}-1}\right|} r^{-d-k^{\prime}} h_{d}
$$

Proof. Stein [St, p.73].

Remark 5.3. When $d=0$ and $h \equiv 1$, Lemma 5.2 makes the well-known classical assertion

$$
\left|\mathbf{S}^{k^{\prime}-1}\right| \mathcal{F}\left(r^{-k}\right)=\left|\mathbf{S}^{k-1}\right| r^{-k^{\prime}} .
$$

When $p=0$, Theorem 5.1 above reduces to precisely the same assertion, because the projections $\Pi$ and $\Phi$ reduce to the identity and zero operators respectively in that case, as noted in $\S 2$.

Our next lemma handles the non-triviality of $\Pi$ and $\Phi$ when $p>0$. Recall that they are the operator fields on $\mathbf{R}^{n}$ which, at each non-zero $x \in \mathbf{R}^{n}$, project $\bigwedge_{*} \mathbf{R}^{n}$ to $\bigwedge_{*} H_{x}$ and $x \wedge \bigwedge_{*} H_{x}$ respectively (cf. (2.2.4)). The wedge operation and its adjoint (2.2.2) yield an elegant and useful pair of formulae for these operations:

Observation 5.4. When $0 \neq x \in \mathbf{R}^{n}$, and $\alpha \in \bigwedge_{*} \mathbf{R}^{n}$, we have

$$
\begin{aligned}
& |x|^{2} \Pi_{x}(\alpha)=(x \wedge \alpha) \vee x, \\
& |x|^{2} \Phi_{x}(\alpha)=x \wedge(\alpha \vee x) .
\end{aligned}
$$

Proof. Extend $e_{1}:=x /|x|$ to an orthonormal basis $\left\{e_{1}, \ldots, e_{n}\right\}$ for $\mathbf{R}^{n}$. It then suffices to check both formulae on the corresponding multivector basis $e_{\lambda}$ defined by (2.2.1), an easy exercise.

We call an operator field $H: \mathbf{R}^{n} \rightarrow \operatorname{Hom}\left(\bigwedge_{*} \mathbf{R}^{n}\right)$ harmonic if

$$
\Delta\langle H(\alpha), \beta\rangle=0
$$

for all constant elements $\alpha, \beta \in \bigwedge_{*} \mathbf{R}^{n}$.

Lemma 5.5. Let $0 \leq p \leq n$. Then there exists a homogeneously quadratic and harmonic operator field $H$ such that, as operators on p-forms, we have

$$
\begin{aligned}
& \Pi=\left(p^{\prime} / n\right) I+r^{-2} H, \\
& \Phi=(p / n) I-r^{-2} H .
\end{aligned}
$$

Here $I$ is the identity on $\bigwedge_{p} \mathbf{R}^{n}$ and $p^{\prime}=n-p$.

Proof. Since $\Pi+\Phi=I$, it suffices to prove the first identity above, or equivalently, that

with $H$ harmonic.

$$
n r^{2} \Pi=p^{\prime} r^{2} I+n H
$$

Observation 5.4 says, for any $\alpha \in \bigwedge_{p} \mathbf{R}^{n}$, that

$$
n r^{2} \Pi(\alpha)=n(x \wedge \alpha) \vee x,
$$

showing that $n r^{2} \Pi$ is polynomially quadratic. Since the ring of homogeneous quadratic polynomials on $\mathbf{R}^{n}$ splits as $r^{2} \mathbf{C}+\mathcal{H}^{2}$, where $\mathcal{H}^{2}$ denotes the harmonic subspace, we can write

$$
n r^{2} \Pi=r^{2} A+n H,
$$


with $A$ constant, and $H \in \mathcal{H}^{2}$. Apply the Laplacian to this expansion and divide by $n$ to get

$$
\Delta\left(r^{2} \Pi\right)=2 A
$$

On the other hand, Observation 5.4 gives, for any basis $p$-vector of the form $e_{\lambda}:=e_{\lambda_{1}} \wedge e_{\lambda_{2}} \wedge \cdots \wedge e_{\lambda_{p}}$ (cf. (2.2.1) $)$

$$
r^{2} \Pi_{x}\left(e_{\lambda}\right)=\sum_{i, j} x_{i} x_{j}\left(e_{i} \wedge e_{\lambda}\right) \vee e_{j} .
$$

Since $\Delta\left(x_{i} x_{j}\right)=2 \delta_{i j}$, and

$$
\left(e_{i} \wedge e_{\lambda}\right) \vee e_{i}= \begin{cases}e_{\lambda}, & i \notin \lambda \\ 0, & i \in \lambda\end{cases}
$$

we see that

$$
\Delta\left(r^{2} \Pi\left(e_{\lambda}\right)\right)=2 \sum_{i}\left(e_{i} \wedge e_{\lambda}\right) \vee e_{i}=2 \sum_{i \notin \lambda} e_{\lambda}=2 p^{\prime} e_{\lambda}
$$

Since the $e_{\lambda}$ form a basis for $\bigwedge_{p} \mathbf{R}^{n}$, this means $\Delta\left(r^{2} \Pi\right)=2 p^{\prime} I$, and comparison with (5.5.2) then yields $A=p^{\prime} I$, turning (5.5.1) into the identity we sought.

Proof of Theorem 5.1. To compute the Fourier transform of $r^{-k} \Pi$, first expand, using the lemma just proven, to get

$$
r^{-k} \Pi=\frac{p^{\prime}}{n} \frac{I}{r^{k}}+\frac{H}{r^{k+2}} .
$$

Since $I$ and $H$ are harmonic, and homogeneous of degree 0 and 2 respectively, Lemma 5.2 computes the Fourier transform of this expansion as

$$
\mathcal{F}\left(r^{-k} \Pi\right)=\frac{p^{\prime}}{n} \frac{\left|\mathbf{S}^{k-1}\right|}{\left|\mathbf{S}^{k^{\prime}-1}\right|} \frac{I}{r^{k^{\prime}}}-\frac{\left|\mathbf{S}^{k+1}\right|}{\left|\mathbf{S}^{k^{\prime}+1}\right|} \frac{H}{r^{k^{\prime}+2}} .
$$

To simplify this, recall that in terms of the classical Gamma function, one has

$$
\left|\mathbf{S}^{k+1}\right|=\frac{2 \pi^{\frac{k}{2}+1}}{\Gamma\left(\frac{k}{2}+1\right)}
$$

for any dimension $k$. But $\Gamma(s+1)=s \Gamma(s)$, implying the recursive identity

$$
\left|\mathbf{S}^{k+1}\right|=\frac{2 \pi}{k}\left|\mathbf{S}^{k-1}\right|,
$$

which reduces (5.5.3) to

$$
\mathcal{F}\left(r^{-k} \Pi\right)=\frac{\left|\mathbf{S}^{k-1}\right|}{\left|\mathbf{S}^{k^{\prime}-1}\right|} \frac{1}{r^{k^{\prime}}}\left(\frac{p^{\prime}}{n} I-\frac{k^{\prime}}{k} \frac{H}{r^{2}}\right) .
$$


Now use the first identity in Lemma 5.5 to rewrite $H / r^{2}$ in terms of $\Pi$ and $I$, to get

$$
\begin{aligned}
\frac{p^{\prime}}{n} I-\frac{k^{\prime}}{k} \frac{H}{r^{2}} & =\frac{p^{\prime}}{n} I-\frac{k^{\prime}}{k}\left(\Pi-\frac{p^{\prime}}{n} I\right) \\
& =\frac{p^{\prime}}{n}\left(1+\frac{k^{\prime}}{k}\right) I-\frac{k^{\prime}}{k} \Pi \\
& =\frac{p^{\prime}}{k} I-\frac{k^{\prime}}{k} \Pi \\
& =\frac{p^{\prime}}{k}(\Pi+\Phi)-\frac{k^{\prime}}{k} \Pi \\
& =\frac{(k-p) \Pi+(n-p) \Phi}{k},
\end{aligned}
$$

since $p^{\prime}-k^{\prime}=k-p$. Inserting this into (5.5.4), we obtain the desired formula for $\mathcal{F}\left(r^{-k} \Pi\right)$.

The subsequent formula for its inverse now follows immediately when $p<k$, because $\Pi$ and $\Phi$ project onto orthogonal complements, so that $\Pi \Phi=\Phi \Pi=0$ and $\Pi+\Phi=I$.

\section{INVERSION, SUPERPOSITION, AND RECONSTRUCTION FROM PROJECTIONS}

Finally, we will combine the convolution formula (Prop. 4.5) with our computation of $\mathcal{F}\left(r^{-k} \Pi\right)$ (Thm. [5.1) to invert $\mathcal{R}_{k}$ explicitly on the space of Schwartz $p$-forms.

When $p=0$, our formula reduces to the well-known inversion of the classical $k^{\prime}$-plane transform. The latter result applies well beyond the Schwartz class, to all continuous functions $\left[\mathrm{H}\right.$, Thm. 6.2] (or even functions in $L_{\mathrm{loc}}^{q}$, with $1 \leq q \leq n / k^{\prime}$ [R, Thm. 4.1]), with $O\left(r^{-n-\varepsilon}\right)$ decay.

We expect that, likewise, our inversion formula holds for $p$-forms with $O\left(r^{-n-\varepsilon}\right)$ decay, leading to both a superposition formula, and an algorithm for reconstructing currents from their projections. We will state the results we anticipate along those lines. We leave their proofs, however, to colleagues more expert in the analytical techniques they seem to require.

Theorem 6.1 (Inversion). Suppose $k<n$, and $\alpha$ is a Schwartz $p$-form on $\mathbf{R}^{n}$, with $p<k$. Then we may recover $\alpha$ from its transform $\mathcal{R}_{k} \alpha$ as follows:

$$
\alpha=C_{n, k, p} \mathcal{F}^{-1}\left(r^{k^{\prime}}\left(\frac{\Pi}{k-p}+\frac{\Phi}{n-p}\right) \cdot \mathcal{F}\left(\mathcal{R}_{k}^{*} \mathcal{R}_{k} \alpha\right)\right) .
$$

Here $\mathcal{F}$ and $\mathcal{F}^{-1}$ apply in the tempered distributional sense, and

$$
C_{n, k, p}=k \frac{\left|\mathbf{S}^{n-1}\right| \mathrm{Ch}(n-1, p)}{\left|\mathbf{S}^{k-1}\right| \operatorname{Ch}(n-k, p)} .
$$

Proof. Apply the convolution formula (Prop. 4.5) to get

$$
\mathcal{R}_{k}^{*} \mathcal{R}_{k} \alpha=c_{1}\left(r^{-k} \Pi\right) \star \alpha,
$$

where

$$
c_{1}=\frac{\left|\mathbf{S}^{k^{\prime}-1}\right| \operatorname{Ch}\left(k^{\prime}, p\right)}{\left|\mathbf{S}^{n-1}\right| \operatorname{Ch}(n-1, p)} .
$$


As noted at the start of $95, r^{-k} \Pi$ represents a tempered $\operatorname{Hom}\left(\bigwedge_{*} \mathbf{R}^{n}\right)$-valued distribution on $\mathbf{R}^{n}$. The multiplication rule for $\mathcal{F}$ therefore extends to the convolution above (see Observation 7.2 for a proof), and we can write its Fourier transform as

$$
\mathcal{F}\left(\mathcal{R}_{k}^{*} \mathcal{R}_{k} \alpha\right)=c_{1} \mathcal{F}\left(r^{-k} \Pi\right) . \mathcal{F}(\alpha)
$$

Theorem 5.1 computes $\mathcal{F}\left(r^{-k} \Pi\right)$ explicitly as an operator field and shows that the right-hand side above, though a priori just a tempered distribution, is actually represented by a locally integrable $p$-form. We can then multiply both sides by the field pointwise inverse to that $p$-form (also given by Theorem 5.1) to deduce

$$
\mathcal{F}(\alpha)=\frac{c_{2}}{c_{1}} r^{k^{\prime}}\left(\frac{\Pi}{k-p}+\frac{\Phi}{n-p}\right) \cdot \mathcal{F}\left(\mathcal{R}_{k}^{*} \mathcal{R}_{k} \alpha\right),
$$

where

$$
c_{2}=c_{2}(n, k):=k \frac{\left|\mathbf{S}^{k^{\prime}-1}\right|}{\left|\mathbf{S}^{k-1}\right|} .
$$

The Fourier transform preserves Schwartz spaces, so $\mathcal{F}(\alpha)$, like $\alpha$ itself, belongs to $\mathcal{S}_{p}\left(\mathbf{R}^{n}\right)$. In particular, the right side of the formula above lies in $\mathcal{S}_{p}\left(\mathbf{R}^{n}\right)$, and we can therefore recover $\alpha$ from $\mathcal{R}_{k} \alpha$ by applying the inverse Fourier transform:

$$
\alpha=\frac{c_{2}}{c_{1}} \mathcal{F}^{-1}\left(r^{k^{\prime}}\left(\frac{\Pi}{k-p}+\frac{\Phi}{n-p}\right) \cdot \mathcal{F}\left(\mathcal{R}_{k}^{*} \mathcal{R}_{k} \alpha\right)\right) .
$$

Since $c_{2} / c_{1}=k C_{n, k, p}$, this completes the proof.

When the codimension $k^{\prime}=n-k$ is even, the unwieldy formula above takes a simpler "differential" form, using the operators $d$ and $\delta$ instead of the Fourier transform:

Corollary 6.2. When the hypotheses of Theorem 6.1 hold with codimension $k^{\prime}=$ : $2 l \in 2 \mathbf{Z}$, we can recover $\alpha$ from $\mathcal{R}_{k} \alpha$ via

$$
\alpha=C_{n, k, p}\left(\frac{-1}{4 \pi^{2}}\right)^{l}\left(\frac{(\delta d)^{l}}{k-p}+\frac{(d \delta)^{l}}{n-p}\right)\left(\mathcal{R}_{k}^{*} \mathcal{R}_{k} \alpha\right) .
$$

Proof. By the formulae in Proposition 2.9 $\mathcal{F}$ intertwines $d \delta$ and $\delta d$ with multiplication by $r^{2} \Phi$ and $r^{2} \Pi$, respectively. But $\Phi$ and $\Pi$ are projections, so $\Phi^{l}=\Phi$ and $\Pi^{l}=\Pi$ for any integer $l>0$, whereby

$$
\begin{aligned}
& \mathcal{F}^{-1} r^{2 l} \Phi \mathcal{F}=\left(\mathcal{F}^{-1} r^{2} \Phi \mathcal{F}\right)^{l}=\left(-\frac{d \delta}{4 \pi^{2}}\right)^{l}, \\
& \mathcal{F}^{-1} r^{2 l} \Pi \mathcal{F}=\left(\mathcal{F}^{-1} r^{2} \Pi \mathcal{F}\right)^{l}=\left(-\frac{\delta d}{4 \pi^{2}}\right)^{l} .
\end{aligned}
$$

When $k^{\prime}=2 l$, these facts simplify our inversion formula in an obvious way. Remark 4.8 then ensures that $\mathcal{R}_{k}^{*} \mathcal{R}_{k} \alpha$ is suitably differentiable, and the corollary follows.

Remark 6.3. The inversion and superposition results above all correctly reproduce their classical scalar precedents when $p=0$, since $\Pi$ is the identity and $\Phi=0$ on $\bigwedge_{0}\left(\mathbf{R}^{n}\right)$. Indeed, one easily checks that these facts reduce the inversion formula of Theorem 6.1 to

$$
f=\frac{\left|\mathbf{S}^{n-1}\right|}{\left|\mathbf{S}^{k-1}\right|} \mathcal{F}^{-1}\left(r^{k^{\prime}} \mathcal{F}\left(\mathcal{R}_{k}^{*} \mathcal{R}_{k} f\right)\right)
$$


for any Schwartz function $f$. For functions, $\delta d=0$ and $d \delta$ is the Laplace operator $\Delta$. So when $k^{\prime}=2 l$ is even and $p=0$, Corollary 6.2 reduces to

$$
f=\left(\frac{-1}{4 \pi^{2}}\right)^{l} \frac{\left|\mathbf{S}^{n-1}\right|}{\left|\mathbf{S}^{k-1}\right|} \Delta^{l}\left(\mathcal{R}_{k}^{*} \mathcal{R}_{k} f\right),
$$

in agreement with $[\underline{\mathrm{H}}$, Thm. 6.2].

Indeed, when $p=0$ the inversion formula extends beyond $\mathcal{S}_{p}\left(\mathbf{R}^{n}\right)$ to (for instance) the class of all continuous functions $\alpha$ with $O\left(r^{-n-\varepsilon}\right)$ decay near infinity, as mentioned above, and we expect the analogous extension to hold for $p>0$ :

Conjecture 6.4. The inversion formulae of Theorem 6.1 and Corollary 6.2 hold for all continuous $p$-forms $\alpha$ with $O\left(r^{-n-\varepsilon}\right)$ decay near infinity for some $\varepsilon>0$.

Now recall again that when $\alpha \in \mathcal{S}_{p}\left(\mathbf{R}^{n}\right)$, so is $\mathcal{F} \alpha$. This fact makes it easy to show that

$$
\phi:=\mathcal{F}^{-1}\left(r^{k^{\prime}}\left(\frac{\Pi}{k-p}+\frac{\Phi}{n-p}\right) \cdot \mathcal{F} \alpha\right)
$$

is smooth, with an $O\left(r^{-n}\right)$ decay estimate. In particular, $\phi$ is integrable on any $k^{\prime}$-plane, so that

$$
\beta:=C_{n, k, p} \mathcal{R}_{k} \phi
$$

(with $C_{n, k, p}$ as in Theorem 6.1) is well-defined and smooth on $\Gamma_{n, k}$. A slightly better $O\left(r^{-n-\varepsilon}\right)$ decay bound for $\phi$ would combine with our conjecture above and our inversion formula to let us write

$$
\phi=C_{n, k, p} \mathcal{F}^{-1}\left(r^{k^{\prime}}\left(\frac{\Pi}{k-p}+\frac{\Phi}{n-p}\right) \cdot \mathcal{F}\left(\mathcal{R}_{k}^{*} \mathcal{R}_{k} \phi\right)\right) .
$$

But the operator

$$
\mathcal{F}^{-1}\left(r^{k^{\prime}}\left(\frac{\Pi}{k-p}+\frac{\Phi}{n-p}\right)\right) \cdot \mathcal{F}
$$

is clearly invertible, and hitting both sides of (6.4.2) with its inverse, given the definition of $\phi$ in (6.4.1), would then give $\alpha=\mathcal{R}_{k}^{*} \beta$, making $\alpha$ a superposition of $k$-planar $p$-forms. So if Conjecture 6.4 holds, so does

Conjecture 6.5 (Superposition). Given $0 \leq p<k<n$, any $\alpha \in \mathcal{S}_{p}\left(\mathbf{R}^{n}\right)$ can be constructed as a superposition of $k$-planar $p$-forms by a formula of the type

$$
\alpha=C_{n, k, p} \mathcal{R}_{k}^{*} \mathcal{R}_{k} \phi,
$$

where $\phi$ is given by (6.4.1) above.

As we have noted, this has been proven when $p=0([\underline{\mathrm{H}}$, Thm. 6.2]). In view of Remark 3.10, it then analyzes the scalar-valued Schwartz function $\alpha$ as a superposition of " $k$ '-plane waves," i.e., functions invariant with respect to the translations generated by $k^{\prime}$ independent vectors. That fact accounts for the classical use of Radon transform methods to solve certain linear partial differential equations by the "method of spherical means" (cf. $[\mathrm{H}, \S 7 \mathrm{a}]$ and $[\underline{\mathrm{R}},(1.1)]$ ).

Conjecture 6.5 would similarly let us analyze Schwartz $p$-forms as superpositions of $k$-planar forms. This in turn would allow us to reconstruct $p$-currents from their projections.

To explain this, we introduce the linear space $\mathcal{E}^{p}\left(\mathbf{R}^{n}\right)$ comprising all smooth $p$-forms on $\mathbf{R}^{n}$. One topologizes $\mathcal{E}^{p}\left(\mathbf{R}^{n}\right)$ in a standard way, so that a sequence in $\mathcal{E}^{p}\left(\mathbf{R}^{n}\right)$ converges to zero if and only if its members, together with all their 
derivatives, converge uniformly to zero on any fixed compact subset of $\mathbf{R}^{n}$ (cf. $[\mathrm{H}$, Chap. V]).

Definition 6.6 (Currents). We call any continuous linear functional on $\mathcal{E}^{p}\left(\mathbf{R}^{n}\right)$ a compactly supported $p$-current on $\mathbf{R}^{n}$.

One easily shows that any such current actually does have compact support, in the sense that for some $R>0$, it vanishes on all smooth $p$-forms supported outside the ball $B_{R}(0)$. Since we can find a Schwartz $p$-form that agrees, on $B_{R}(0)$, with any given smooth $p$-form, it follows immediately that

Observation 6.7. A compactly supported $p$-current is determined by its values on $\mathcal{S}_{p}\left(\mathbf{R}^{n}\right) \subset \mathcal{E}^{p}\left(\mathbf{R}^{n}\right)$.

Finally, note that we can map any such current $T$ into a $k$-plane $P \in \mathrm{G}_{n, k}$, to produce a compactly supported $k$-current called the projection of $T$ into $P$, and denoted by $P_{*} T$, via the simple rule

$$
P_{*} T[\phi]:=T\left[P^{*} \phi\right] \text { for all } \phi \in \mathcal{E}^{p}(P) .
$$

Here, as in our introduction, $P^{*} \phi$ denotes the pullback of $\phi$ from $P$ to $\mathbf{R}^{n}$ via the orthogonal projection $\mathbf{R}^{n} \rightarrow P$.

Conjecture 6.8. When $p<k<n$, each compactly supported $p$-current $T$ on $\mathbf{R}^{n}$ is uniquely determined by its projections into all $k$-planes $P \in \mathrm{G}_{n, k}$. Explicitly, for any $\alpha \in \mathcal{S}_{p}\left(\mathbf{R}^{n}\right)$, we have

$$
T[\alpha]=\int_{G n, k} P_{*} T[\beta(P, \cdot)] d P,
$$

where $\beta$ is the mapping constructed from $\alpha$ in Theorem 6.5 .

Proof (modulo Conjecture 6.4). By Observation 6.7, it suffices to show that whenever $\alpha \in \mathcal{S}_{p}\left(\mathbf{R}^{n}\right)$, we can determine $T[\alpha]$ from the projections $P_{*} T$ for every $P \in \mathrm{G}_{n, k}$. But if Conjecture 6.4 holds, then so does Conjecture 6.5, and the latter constructs a $p$-form $\phi$ from $\alpha$ for which the mapping $\beta:=\mathcal{R}_{k} \phi$ satisfies

$$
\alpha(x)=\left(\mathcal{R}_{k}^{*} \beta\right)(x)=\left.\int_{\mathrm{G}_{n, k}} \beta(P, P x)\right|_{P} d P
$$

for every $x \in \mathbf{R}^{n}$. Since $\beta$ lies in the image of $\mathcal{R}_{k}$, Remark 3.4 makes the restriction $\left.\right|_{P}$ superfluous in the formula above, which we can thus rewrite as

$$
\alpha=\int_{\mathrm{G}_{n, k}} P^{*}(\beta(P, \cdot)) d P .
$$

It would then follow that

$$
\begin{aligned}
T[\alpha] & =T\left[\int_{\mathrm{G}_{n, k}} P^{*}(\beta(P, \cdot)) d P\right] \\
& =\int_{\mathrm{G}_{n, k}} T\left[P^{*}(\beta(P, \cdot))\right] d P \\
& =\int_{\mathrm{G}_{n, k}} P_{*} T[\beta(P, \cdot)] d P,
\end{aligned}
$$

proving the result. 


\section{Appendix: Technical lemmas}

To clarify the main logic of our exposition, we have deferred two technical facts about convolution to this appendix.

We start by verifying that the notion of convolution extends to the setting required by our inversion theorem.

Observation 7.1. When $F \in \mathcal{S}\left(\mathbf{R}^{n}, V\right)$, the classical definition of $T \star F$ (see Item (3) in (2.7) extends in a natural way to the case where $T$ is a tempered $(V, W)$-distribution.

Proof. To do this, we identify vectors with operators in a somewhat unusual way.

Let $v^{*}$ denote the image of $v \in V$ under the canonical duality $V \sim V^{*}$. We then let $v_{\otimes}: W \rightarrow \operatorname{Hom}(V, W)$ signify the operator we obtain by setting

$$
\left(v_{\otimes}(w)\right)(x):=\left(w \otimes v^{*}\right)(x):=v^{*}(x) w \in W
$$

for all $x \in V$ and $w \in W$. We can then identify any $V$-field $F$ on $\mathbf{R}^{n}$ with the field $F_{\otimes}$ given by

$$
x \mapsto F_{\otimes}(x):=F(x)_{\otimes} .
$$

A routine calculation shows that whenever $T \in \mathcal{S}\left(\mathbf{R}^{n}, V, W\right), F \in \mathcal{S}\left(\mathbf{R}^{n}, V\right)$ and $G \in \mathcal{S}\left(\mathbf{R}^{n}, W\right)$, we have

$$
(T . F)[G]=T\left[G \otimes F^{*}\right] .
$$

Using this fact with a bit more calculation then reveals that

$$
(T \star F)[G]=T\left[F_{\otimes}^{-} \star G\right],
$$

where the superscript "_" indicates reflection through the origin, e.g.,

$$
F^{-}(x):=F(-x) \text {. }
$$

Since $F_{\otimes}^{-} \star G$ lies in $\mathcal{S}\left(\mathbf{R}^{n}, V, W\right)$, (7.1.2) now defines $T \star F$ for any $T \in$ $\mathcal{S}^{*}\left(\mathbf{R}^{n}, V, W\right)$, as promised.

We next show that the Fourier convolution rule (Item (3) of $\$ 2.7$ again) extends to convolutions of the type just described:

Observation 7.2. The Fourier convolution/product rule

$$
\mathcal{F}(T \star F)=\mathcal{F}(T) . \mathcal{F}(F)
$$

holds when $T \in \mathcal{S}^{*}\left(\mathbf{R}^{n}, V, W\right)$ and $F \in \mathcal{S}\left(\mathbf{R}^{n}, V\right)$.

Proof. In view of (17.1.1) and (2.7.2), we have to show that for any $G \in \mathcal{S}\left(\mathbf{R}^{n}, W\right)$,

$$
(T \star F)[\mathcal{F}(G)]=T\left[\mathcal{F}\left(G \otimes \mathcal{F}(F)^{*}\right)\right] .
$$

We start by using (7.1.2) to write

$$
(T \star F)[\mathcal{F}(G)]=T\left[F_{\otimes}^{-} \star \mathcal{F}(G)\right] .
$$

Since $F$ and $G$ are both Schwartz, properties (1), (2) and (especially) the convolution/product rule (3) listed above, together show that

$$
\begin{aligned}
F_{\otimes}^{-} \star \mathcal{F}(G) & =\mathcal{F}^{-1}\left(\mathcal{F}\left(F_{\otimes}^{-}\right) \cdot \mathcal{F}^{2}(G)\right) \\
& =\mathcal{F}\left(\mathcal{F}(F)_{\otimes}^{-} \cdot G^{-}\right)^{-} \\
& \left.=\mathcal{F}\left(G \otimes \mathcal{F}(F)^{*}\right)\right) .
\end{aligned}
$$

The desired fact (7.2.1) follows immediately. 


\section{REFERENCES}

[Fe] H. Federer, Geometric Measure Theory, Springer-Verlag, Berlin, Heidelberg, 1969. MR 0257325 (41:1976)

[Fo] G. B. Folland, Introduction to Partial Differential Equations, Princeton University Press, Princeton, NJ, 1978. MR.1357411 (96h:35001)

[Fu] B. Fuglede, An integral formula, Math. Scand., 6 (1958), 207-212. MR0105724 (21:4460)

[H] S. Helgason, The Radon Transform, 2nd ed. Progress in Mathematics, 5. Birkhäuser Boston, Inc., Boston, MA, 1999, ISBN: 0-8176-4109-2. MR.1723736 (2000m:44003)

[GGG] I. M. Gelfand, S. G. Gindikin, and M. I. Graev, Problems of integral geometry connected with the integration of differential forms over straight lines in $R^{3}$ and $\mathbf{C}^{3}$ (Russian, English summary) Akad. Nauk SSSR Inst. Prikl. Mat. Preprint No. 24 (1979) (42 pp.) MR542289 (82g:53077)

[GGS] M. I. Gelfand, I. M. Graev, and Z. Ya. Shapiro, Differential forms and integral geometry, (Russian) Funkcional. Anal. i Prilozhen. 3 (1969), no. 2, 24-40. MR0244919 (39:6232)

[R] B. Rubin, Reconstruction of functions from their integrals over $k$-planes, Israel J. Math., 141 (2004), 93-117. MR2063027 (2005b:44004)

[St] E. Stein, Singular Integrals and Differentiability Properties of Functions, Princeton Mathematical Series 30, Princeton Univ. Press, 1979. MR0290095 (44:7280)

[Str] R. Strichartz, A Guide to Distribution Theory and Fourier Transforms, World Scientific Publishing Co., 2003. MR2000535

Department of Mathematics, Indiana University, Bloomington, Indiana 47405

E-mail address: solomon@indiana.edu

$U R L$ : mypage.iu.edu/ solomon 